\title{
The Principle of Full FunCtionality - the BaSis FOR RAPID RECONFIGURATION IN HETEROGENEOUS MODUlaR MOBILE ROBOTS
}

\author{
Victor Andreev, Valerii Kim \& Pavel Pletenev
}
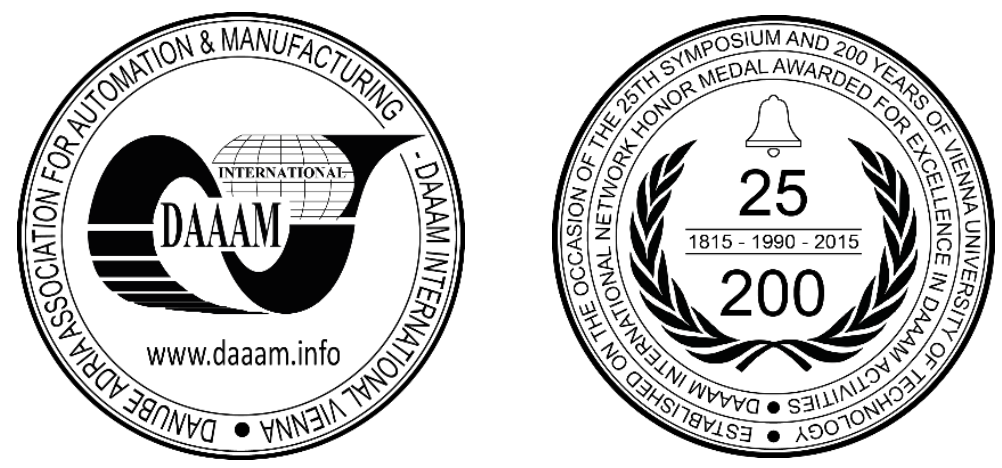

This Publication has to be referred as: Andreev, V[iktor]; Kim, V[alerii] \& Pletenev, P[avel] (2017). The Principle of Full Functionality - the Basis for Rapid Reconfiguration in Heterogeneous Modular Mobile Robots, Proceedings of the 28th DAAAM International Symposium, pp.0023-0028, B. Katalinic (Ed.), Published by DAAAM International, ISBN 978-3-902734-11-2, ISSN 1726-9679, Vienna, Austria

DOI: $10.2507 / 28$ th.daaam.proceedings.003

\begin{abstract}
This paper proposes to design a heterogeneous modular mobile robot control system based on the principle of full functionality of the modules. The full functionality of a mechatronic device is the ability to perform its goal function using only its own facilities for executing instructions from an external control system. The external control system is a module-supervisor of modular robot in relation to the other modules of the robot. This module forms the control goal and verifies only the result of its achievement by executive module, but doesn't control the task execution process by the module. The full functionality of robot modules is achieved by designing each module as a full-featured electronic or mechatronic device with its own the information-measuring and control system that containing all necessary components: sensors, computing devices and actuators. The use of a network principle of the robot's information-measuring and control system construction and the Ethernet standards provides the capability of direct inter-module information interaction and the ability to rapidly form and alter the structure of the robot directly on the scene of the works and during the works themselves.
\end{abstract}

Keywords: mobile robot; modular robot; heterogeneous robot; control system; reconfiguration

\section{Introduction}

The maximum uncertainty in terms of future work in case of emergency situations, accidents, catastrophes, and active countermeasures makes it no possible to determine in advance the composition and configuration of necessary robotics [1]. Therefore, mobile robots (MR) that are focused on the use in extreme situations, must possess the ability to rapidly form and alter its structure directly on the works location and during the works themselves. In other words, the robots with variable structure must be used to perform such works, i.e. a reconfigurable modular mobile robots. At now the modular mobile robotics is at an early stage of its development. One of the main difficulties of creating the reconfigurable systems is to develop a plug and play control systems. In such robots, an automatic reorganization of the overall control system structure should be realized in accordance with the rapidly changing composition and configuration of the robotic 
device. Such a requirement leads to a significant increase in the system complexity of the coordinated control of all modules of a heterogeneous robot.

The purpose of the study is to suggest a new structure of the software and hardware part of the modular mobile robot control system, which would be simple enough and possess the ability of automatic reorganization. The use of the principle of the full functionality for the modules design makes it possible to simplify such control system.

The heterogeneous modular mobile robot and its modules are mechatronic devices. The full functionality of a mechatronic device is the ability to perform its goal function using only its own facilities for executing instructions from an external control system. The main control system of a modular robot is a module-supervisor in relation to the other modules of the heterogeneous modular mobile robot. The full functionality of robot modules is achieved by designing each module as a full-featured electronic or mechatronic device with its own the information-measuring and control system (IMCS) that containing all necessary components: sensors, computing devices and actuators.

This functional completeness is the main difference of our solution from the other heterogeneous robots where module-supervisor controls the operation of all modules at the executive level. In our solution the module-supervisor forms the control goal and verifies only the result of its achievement by executive module, but doesn't control the task execution process by the module. As a consequence of this approach, a distributed control is realized. Such distributed control allows parallelizing the computational process of realisation of the robot goal function by dividing the process into the functional subtasks and their distributing among the microprocessors of modules. Thus the module-supervisor computer performs only the following functions: splitting the process into functional sub-tasks, distribution of tasks between the modules, control of their execution, the processes synchronization and the solution of behavioural tasks. The implementation of the functional subtask is performed by the corresponding full-featured electronic or mechatronic robot module. As a result, the module-supervisor computational power requirements are reduced.

Task 1. To implement distributed control it's necessary to develop a language for inter-module communication.

Another problem of creating reconfigurability is to develop means of mechanical, electrical and information interaction between the modules, i.e. the corresponding interfaces. On the one hand, each interface should be simple, and on the another - universal. This will ensure the ease of the robot assembly from the modules developed by different manufacturers in the same way as it is done in computer technology. For example, printers, scanners and other external devices from different manufacturers are easily connected to a computer equipped with an appropriate operating system (OS) and drivers. We believe that the modular robot should be assembled similarly, i.e. on a plug and play method.

Task 2. It is necessary to define the communication environment (electrical interface) and to develop software (software interface) that will provide an assembly of the robot from the modules of different manufacturers in the "plug and play" mode. At this stage of the project the mechanical interface we are not yet considering.

\section{Modern solutions in the modular mobile robotics}

In the course of works of accident consequences elimination at the Chernobyl nuclear power plant (Soviet Union, 26 April 1986), a new direction in modular robotics has been appeared - reconfigurable modular robots.

Currently, modular mobile robots are divided into two types: homogeneous and heterogeneous. In homogeneous robots most of the modules are identical in structure and functionality. Essentially this is a technical analog of colonial organisms. The main objective of such systems is to provide autonomous reconfiguration and adaptation to any environmental conditions. Homogeneous robots are modular robots such as M-TRAN [2] (2002 - 2008), ATRON [3] (2006), Transmote [4] (2012).

Homogeneous modular robots have several unique properties that distinguish them among the diversity of mobile robots. Those properties are as follows:

- adaptation to different and sometimes extreme environmental conditions thanks to automatic reconfiguration;

- high failure resistance of individual modules during execution of work;

- scalability of modular systems.

To the disadvantages of homogeneous robots can be attributed:

- each module should have all equipment for movement and navigation;

- complexity of the coordinated control of a modular system;

- high requirements to the design and control system of the each module;

- system scalability is limited by the capabilities of software and hardware implementation of the modules.

In addition, when structure and functionality of all modules are the same, one module is usually different from the rest this module is needed for a common control and communication with external supervisor.

A distinctive feature of heterogeneous robots is the differentiation of modules according to their functionality. Each module is responsible for its own limited set of tasks, so the modules in heterogeneous systems unequal. The heterogeneous robots can be exemplified by the modular robots such as Thor [5] (2013), SMART [6] (2012), and modular robot for space exploration [7] (2006).

Despite the lower level of autonomy of heterogeneous systems compared to homogeneous systems, many practical problems are solved more efficiently by systems with modules of different functionality. 
Heterogeneous modular robots have the following advantages:

- relatively low requirements to the control system of modules and their design;

- scalability of the robot is practically unlimited;

- the ability to perform a wide range of tasks;

- the ability to adapt to environmental conditions.

To the disadvantages of heterogeneous robots can be attributed:

- the complexity of automatic reconfiguration implementation;

- low failure tolerance of individual modules;

- the complexity of the coordinated control of all modules.

It should be noted that in the control system of considered homogeneous and heterogeneous mobile robots it is observed the presence of elements of distributed control.

From the position of creating mobile robots with variable structure, i.e. reconfigurable modular robots, focused on the use in case of extreme situations, we believe that to date heterogeneous robots have the advantage compared to homogeneous robots. Also we believe that the automatic reconfiguration of robot in general, while solving the aforesaid tasks is not actual, since it can be done manually, but automatic rebuilding of all structures of the robot IMCS to the new composition and configuration needs to be solved necessarily. Low failure resistance of individual modules is usually solved by duplicating the systems and replacement of faulty modules on the same or modified. But such replacement requires the capability to quickly adapt the robot IMCS to its new structure. This leads to increase of the complexity of the coordinated control of all modules. Therefore, the desired solution of the robot IMCS should allow the rapid reconfiguration of the robot.

\section{The principle of the full functionality of modules}

Each module should be a full-featured electronic or mechatronic device with its own the information-measuring and control system. This means that each module of the robot needs to fulfill its mission in any convenient to it way - it follows that the module design must meet the functional purpose of the module, and the module IMCS must fully provide information and executive functionality [8].

Consider the principle of full functionality by the example of the transport module (Fig.1). Let the robot consists of the following modules: module-supervisor, transport module (TM) and sensor modules. Module-supervisor receives from an external supervisor (e.g., human operator) common task. The software of module-supervisor breaks down the task into subtasks and distributes them to the appropriate modules. Module-supervisor sends to TM the control goal - a vector of desired robot position $\left[X_{G}, Y_{G}, \varphi_{G}\right]^{\mathrm{T}}$, where $X_{G}, Y_{G}$ - the robot characteristic point coordinates with respect to the fixed world coordinate frame, $\varphi_{G}$ - the robot orientation angle. The transport module should independently achieve this target location, using the methods of control laid down in its control system, and information from its sensory systems.

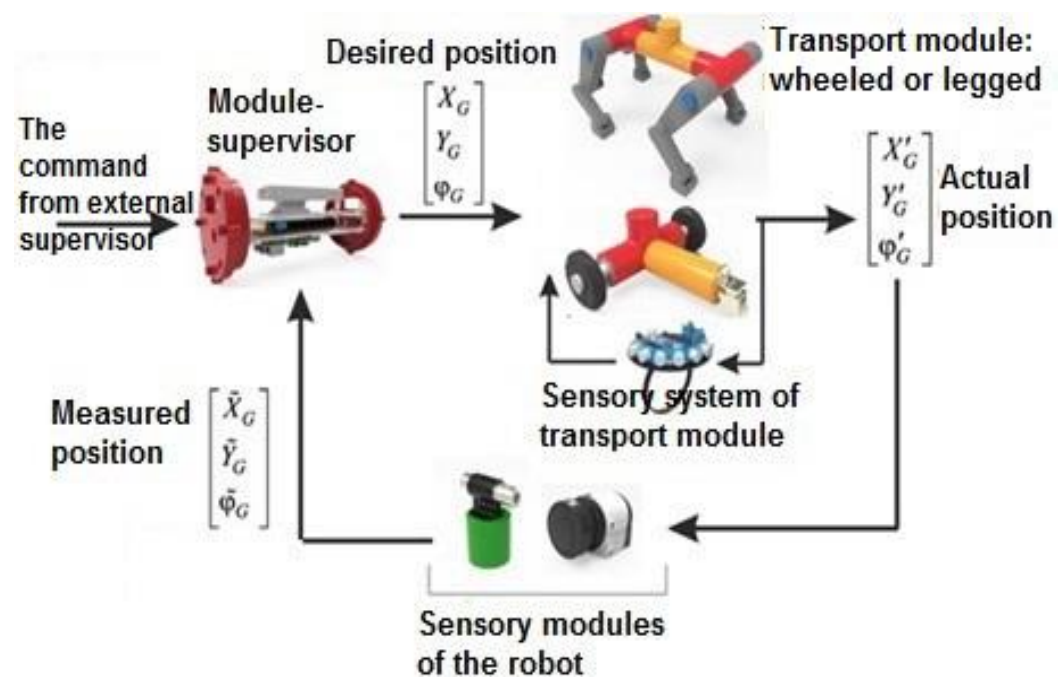

Fig. 1. The principle of full functionality by the example of the transport module

The TM sensor system can function as a separate module with a limited range of operation (for example, in the range of $5 \div 100 \mathrm{~cm}$ ) and have information communication only with the computer of the transport module, i.e. the sensor system is a part of the transport module IMCS [9]. Due to the natural position errors of trajectory following by the transport module, its actual position, and therefore the position of whole robot, specified by the vector $\left[X_{G}^{\prime}, Y_{G}^{\prime}, \varphi_{G}^{\prime}\right]^{T}$, will differ from the desired position. Here, the accuracy of achievement of the control goal is tested by the module-supervisor with the help of sensor modules of the robot. A sensor modules of the robot that use more sophisticated sensors (television, ultrasonic, infrared, etc.), provide affixment of the robot to the fixed world coordinate frame, for example, from landmarks 
in the environment. Comparing the vector of measured position $\left[\tilde{X}_{G}, \tilde{Y}_{G}, \tilde{\varphi}_{G}\right]^{T}$ with desired, the module-supervisor if necessary, corrects the actual position of the robot, setting a new goal.

In this scheme, the transport module may be implemented as the wheeled, legged robot, or any other mobile platform, but it must accomplish the control goal given by a module-supervisor, regardless of its mechanical construction, the structure of the IMCS and the control algorithm of the module.

The distributed control based on the principle of full functionality is implemented. Such distributed control allows parallelizing the computational process of the robot target function executing by dividing the process into the functional subtasks and their distributing among the microprocessors of modules. Then the computer of module-supervisor performs only the following functions: splitting the process into functional sub-tasks, distribution of tasks between the modules, control of their execution, processes synchronization and the solution of behavioral tasks. In addition, the modulesupervisor carries out information interaction with the external supervisor, for example, the operator of a mobile robot.

To implement such distributed control it's necessary to develop a language of inter-module communication and software that supports dynamic reconfiguration of the robot IMCS.

\section{The inter-module communication}

Modern modular robots (homogeneous and heterogeneous) for inter-module communication, as a rule, use different serial bus: I2C - modules of RTC; CAN - in robots M-TRAN, SMART; IrDA (essentially RS-232) - ATRON; RS-485 - in robots Thor, Odin. Serial bus is used on the robot TRANSMOTE - it uses ZigBee wireless interface. In such systems the role of inter-module interaction is to ensure the most rapid and effective transfer of commands to the executive or tactical level from an external computer to the modules, and transmission of sensor data. In this case, the information flow in the process of inter-module interaction is small, because on each module there are no sensors or many sensors cannot be installed. As a result, it is necessary basically to transmit only the executive level commands.

Our solution involves the use of a duplex sensor system (sensor modules near and far radius of action) with a large variety of sensors, including multi-camera vision system. In this case, the system inter-module communication must have a high throughput. Listed interfaces do not have sufficient bandwidth. It is proposed to organize inter-module communication via the interface, developed on the basis of Ethernet. It is a broadband, reliable and affordable interface. Its modification EtherCAT (for real-time) does not allow the creation of horizontal linkages - all inter-module communication must be through the "master" bus, which eliminates the possibility of direct inter-module interaction. Since the use of the principle of full functionality of modules eliminates the need to transfer commands of the executive level, as noted previously, it is allowed more soft timing requirements of message delivery and the use of interfaces with the "soft real time". In our case, all inter-module communication is restricted to small dimensions of the robot, so we choose the topology of the network of type "star", which corresponds to a typical Ethernet network topology.

Currently, for control of dissimilar mechatronic devices the software framework ROS (Robotics Operating System) is widely used. However, a direct application of ROS is "heavy" - you need at least a computer running a full Linux OS, you must additionally set a large set of various software, which requires a high level of input use (for example, dialects of XML are using for the configuration). In the modules of the robot the microcontrollers or, as a maximum, single board computers are typically used; ROS is not focused to work directly on the microcontroller [10]. Therefore, we must create a software framework that would be compatible with ROS, but it would be easy to implement on embedded systems.

The choice of the Ethernet standard allows realizing the modular robot information-measuring and control system in the form of local area network, the nodes of which are IMCS of individual modules [11]. This solution allows transferring such network properties as scalability and reconfiguration to the network structure of the robot's IMCS. Experience in the development and long-term exploitation of the network training Internet laboratory convinces authors in the correctness of the choice made [12]. The method of geographically distributed control designed for the laboratory forms the basis of the method of inter-module information interaction.

The approach proposed in [12] is based on the library Zmq_robot [13], which was based on ZMQ library. Library Zmq_robot allows you to create software as for modules, and the control programs for a supervisor. However, this library has the same disadvantage that ROS - the complicated software that only runs on a full OS and not working on embedded systems must be installed on the device. To solve this problem the method for specification of inter-module interaction that takes into account the modules full functionality property was developed. For the organization of inter-module interaction we propose to use the ZMQ library as the basis. But in an addition to the ZMQ the UDP protocol broadcast messages, which allows implementing a specification for embedded systems are used. Unlike ROS, in this software each module has a single interface that is common for all modules and provides general information about itself - the module name, its description, functional interfaces, etc. The proposed approach of the inter-module information interaction is described in more detail in [14].

\section{Conclusion}

A new structure of the hardware and software part of the control system of the heterogeneous modular mobile robots is proposed. This structure is fairly simple to implement and has the ability of automatic reorganization. The basis of this structure is the principle of the full functionality of the modules - each module is designed as a full-featured electronic or 
mechatronic device with its own information-measuring and control system. Automatic reconfiguration of the robot control system is achieved by using:

a) a network structure of the information-measuring and control system based on the Ethernet technology, in which each module is a node of the local area network;

b) the developed specification for the method of an inter-module communication, based on the principles of the Robotic Operating System, but implemented in the embedded computing systems;

c) the developed language for an inter-module communication.

This approach provides:

d) parallelizing of the computational process due to its distribution between the calculation devices of the modules, which simplifies the overall control task and reduces the requirements for the modules calculation devices;

e) the ability to quickly assemble a robot from modules of different functional purpose and different manufacturers by using the appropriate drivers.

Currently, these studies are at the most basic level. The use of the principle of full functionality for the design of the IMCS of a heterogeneous modular mobile robot was tested on a laboratory working model consisting of the intelligent control module (module-supervisor), the wheeled transport module, the short-range sensors module and the power supply module (the batteries). The focus at this stage was paid to ensuring the requirements of the full functionality of the transport module and the power supply module. The functions of all modules are implemented in the minimal version. For the transport module, a novel motion planning method for a differential drive mobile robot using two-dimensional vector fields is proposed. It is assumed that the sensor module consists only of odometers, distance sensors and a microcontroller that processes signals from sensors. The robot information-measuring and control system is implemented as a local area network Ethernet. The software for inter-module information interaction is built on the basis of the ZMQ library using the UDP network protocol with broadcast messages.

Experiments have shown the promise of this area of research. In the near future we are going to increase the functional composition of the robot modules, increase the number of remote sensors and their diversity in the short-range sensor module, and also complicate the functionality of the module-supervisor.

We believe that the proposed principle of modular design of robotic systems can be successfully used in the Bionic Assembly System $[15,16]$.

\section{Acknowledgments}

The authors are expressing their sincere gratitude to S. Kuvshinov, Director of International Institute of the New Educational Technologies of Russian State University for the Humanities, for assistance in construction of the experimental equipment.

This work was supported by the Russian Foundation for Basic Research, grants no. 16-07-00811a \& 16-07-01264a.

\section{References}

[1] Lopota, A.V. \& Yurevich, E.I. (2013). Stages and development prospects of robotic systems design modular principle. St. Petersburg State Polytechnical University Journal. Computer Science. Telecommunication and Control Systems. No.1(164), 2013, pp. 98-103, ISSN 1994-2354.

[2] Murata, S.; Yoshida, E.; Kamimura, A.; Kurokawa, H.; Tomita, K. \& Kokaji, S. (2002). M-TRAN: selfreconfigurable modular robotic system. IEEE/ASME Transactions on Mechatronics, n.7(4), 2002, pp.432-441.

[3] Østergaard, E.H.; Kassow, K.; Beck, R. \& Lund, H.H. (2006). Design of the ATRON lattice-based selfreconfigurable robot. Autonomous Robots, No.21(2), 2006, pp.165-183.

[4] Guifang Qiao, Guangming Song, Jun Zhang, Hongtao Sun, Weiguo Wang \& Aiguo Song (2012). Design of Transmote: a Modular Self-Reconfigurable Robot with Versatile Transformation Capabilities. Proceedings of the 2012 IEEE International Conference on Robotics and Biomimetics, pp.1331-1336.

[5] Lyder, A.H.; Stoy, K.; Mendoza-Garcia, R.-F.; Larsen, J.C. \& Hermansen, P. (2013). On sub-modularization and morphological heterogeneity in modular robotics. Intelligent Autonomous Systems, No.12, V.193 of Advances in Intelligent Systems and Computing, Springer Berlin Heidelberg, 2013, pp. 649-661.

[6] Baca, J. ; Ferre, M. \& Aracil, R. (2012). A heterogeneous modular robotic design for fast response to a diversity of tasks. Robotics and Autonomous Systems, vol. 60, no.4, 2012, pp. 522-531.

[7] Hancher, M.D. \& Hornby, G.S. (2006). A modular robotic system with applications to space exploration. 2nd IEEE International Conference on Space Mission Challenges for Information Technology (SMC-IT'06). - Pasadena, CA: Publisher «IEEE», 2006, pp.132-140.

[8] Andreev, V. \& Kim, V. (2017)., The Application of Artificial Vector Fields for Motion Control of a Heterogeneous Modular Mobile Robot, Proceedings of the 28th DAAAM International Symposium, pp.xxxx-xxxx, B. Katalinic 
(Ed.), Published by DAAAM International, ISBN 978-3-902734-xx-x, ISSN 1726-9679, Vienna, Austria, OI: $10.2507 / 28$ th.daaam.proceedings.xxx, in press.

[9] Andreev, V. \& Kim, V. (2016). Control system and design of the motion module of a heterogeneous modular mobile robot, 27th International DAAAM Symposium on Intelligent Manufacturing and Automation 2016, Proceedings of a meeting held 26-29 October 2016, Mostar, Bosnia and Herzegovina, B. Katalinic (Ed.), ISBN 978-1-5108-33005. Curran Associates, Inc., NY 12571 (Jan 2017), pp.0586-0594.

[10] Andreev, V.; Pletenev, P. \& Poduraev, Y. (2015). The method of network control of robotic systems of different models and manufacturers, Proceedings of the 26th DAAAM International Symposium., B. Katalinic (Ed.), Published by DAAAM International, ISBN: 978-1-5108-1839-2, ISSN 1726-9679, Vienna, Austria. 2015. Curran Associates, Inc., New York, Feb. 2016, pp.0727-0730. DOI: 10.2507/26th.daaam.proceedings.101.

[11] Andreev, V. \& Poduraev, Y. (2016). Network-based Design of Heterogeneous Modular Mobile Robotic Systems, 27th International DAAAM Symposium on Intelligent Manufacturing and Automation 2016, Proceedings of a meeting held 26-29 October 2016, Mostar, Bosnia and Herzegovina, B. Katalinic (Ed.), ISBN 978-1-5108-3300-5. Curran Associates, Inc., NY 12571 (Jan 2017), pp.0004-0009.

[12] Andreev, V.; Pryanichnikov, V. \& Kirsanov, K. (2015). Geographically distributed "multi-operator" Control for Mechatronic Devices via the Internet, Proceedings of the 26th DAAAM International Symposium., B. Katalinic (Ed.), Published by DAAAM International, ISBN: 978-1-5108-1839-2, ISSN 1726-9679, Vienna, Austria. 2015. Curran Associates, Inc., New York, Feb. 2016, pp. 0004-0011. DOI: 10.2507/26th.daaam.proceedings.001.

[13] Kirsanov, K. (2014). Software architecture of control system for heterogeneous group of mobile robots, 25th DAAAM Int. Symp. on Intelligent Manufacturing and Automation, 2014. Procedia Engineering, Elsevier BV. ISSN 1877-7058, 2015, V.100, pp.216-221. DOI: 10.1016/j.proeng.2015.01.339.

[14] Andreev, V. \& Pletenev, P. (2017). Organizing Intermodular Communication for Heterogeneous Modular Mobile Robot, Proceedings of the 28th DAAAM International Symposium, pp.xxxx-xxxx, B. Katalinic (Ed.), Published by DAAAM International, ISBN 978-3-902734-xx-x, ISSN 1726-9679, Vienna, Austria, OI: 10.2507/28th.daaam.proceedings.xxx, in press.

[15] Katalinic, B.; Kukushkin, I.; Pryanichnikov, V. \& Haskovic, D. (2014). Cloud Communication Concept for Bionic Assembly System. Procedia Engineering, 1877-7058, 69 (2014), pp.1562-1568 doi:10.1016/j.proeng. 2014.03.156.

[16] Katalinic, B.; Kukushkin, I. \& Haskovic D. (2014). Bionic Assembly System Cloud: Functions, Information Flow and Behavior. In 9th International Conference of DAAAM Baltic, Industrial Engineering, ISBN 978-9949-23-6206, ISSN 2346-6138, pp.103-108, ed. T.Otto, Tallinn, Estonia, 2014. 